\begin{tabular}{|c|c|}
\hline Title & Uncertainty quantification of neutronic parameters of light water reactor fuel cells with JENDL- 4.0 covariance data \\
\hline Author(s) & Chiba, Go; Tsuji, Masashi; Narabay ashi, Tadashi \\
\hline Citation & $\begin{array}{l}\text { Journal of Nuclear Science and Technology, 50(7), 751-760 } \\
\text { https://doi.org/10.1080/00223131.2013.788793 }\end{array}$ \\
\hline Issue Date & 2013-07-01 \\
\hline Doc URL & http:/hdl.handle.net/2115/56470 \\
\hline Rights & $\begin{array}{l}\text { This is an A uthor's A ccepted Manuscript of an article published in Journal of Nuclear Science and Technology } 2013 \odot \\
\text { Taylor \& Francis, avail able online at: http:/Www.tandfonline.com/10.1080/00223131.2013.788793 }\end{array}$ \\
\hline Type & article (author version) \\
\hline File Information & paper.pdf \\
\hline
\end{tabular}

Instructions for use 


\title{
Uncertainty quantification of neutronic parameters of light water reactor fuel cells with JENDL-4.0 covariance data
}

\author{
Go CHIBA* $^{* a}$, Masashi TSUJI ${ }^{a}$ and Tadashi NARABAYASHI ${ }^{a}$ \\ ${ }^{a}$ Graduate School of Engineering, Hokkaido University, Sapporo 060-8628, Japan
}

Neutronic parameter uncertainty induced by nuclear data uncertainty is quantified for several light water reactor fuel cells composed of different combinations of fissile/fertile nuclides. The covariance data given in JENDL-4.0 is used as the nuclear data uncertainty and uncertainty propagation calculations are carried out using sensitivity coefficients calculated with the generalized perturbation theory for burnup-related neutronic parameters.

It is found that main contributors of nuclear data uncertainty to the neutronic parameter uncertainty are uranium-238 capture cross section in a uranium-oxide fuel cell, plutonium-240 and -241 capture cross sections and fission spectrum of fissile plutonium isotopes in a uranium-plutonium mixed-oxide fuel cell. It is also found that thorium-232 capture cross section uncertainty is a dominant source of neutronic parameter uncertainty in thorium-uranium and thorium-plutonium mixed-oxide fuel cells. It should be emphasized that precise and detail information of component-wise uncertainties can be obtained by virtue of the adjoint-based sensitivity calculation methodology. Furthermore, cross-correlations are evaluated for each fuel cell, and strong correlations among the same parameters at BOC and EOC and among different parameters are observed. 
KEYWORDS: uncertainty quantification, JENDL-4.0, covariance data, generalized pertur-

bation theory 


\section{Introduction}

Enormous effort has been made to improve models and methodologies for nuclear data evaluations and reactor physics calculations so far, and consequently quite precise physical and numerical models have been realized. Those enable us to better understand physical behavior of fission reactors, and to drastically improve prediction capability for reactor neutronic parameters.

Even if physical theory and numerical model have been greatly sophisticated, calculated reactor neutronic parameters inevitably have their uncertainties with a certain degree. Uncertainty quantification of the calculated parameters are required in order to assign an appropriate margin to those parameters.

Importance of uncertainty quantification has been well recognized recently in a nuclear engineering field, especially in nuclear data community. The most recent nuclear data library ENDF/B-VII.1 released in 2011 gives uncertainty information (covariance data) to 190 nuclides.[1] With the uncertainty information of the nuclear data, reactor neutronic parameter uncertainty induced by the nuclear data uncertainty can be quantified by uncertainty propagation calculations, such as random sampling calculations, sensitivity-based calculations, etc.

Among several nuclear data libraries, the first one which contains covariance data applicable to uncertainty quantification of reactor neutronic parameters is the former version of Japanese evaluated nuclear data library JENDL-3.3 which was released more than ten years before.[2] JENDL-3.3 has been widely utilized for uncertainty quantification of reactor neutronic parameters of fast reactors and accelarated-driven sub-critical reactors. $[3,4]$ 
In 2010, the new version of JENDL, JENDL-4.0, has been released.[5] The number of nuclides, to which the covariance data is given, is 95 and it is much larger than that of JENDL-3.3. Furthermore, in JENDL-4.0, covariance data in the resonance energy range for important heavy nuclides, such as uranium-235 and plutonium-239, have been obtained from the covariance data simultaneously evaluated in the resonance analysis while those have been evaluated with the retroactive method in JENDL-3.3. After the release of JENDL-4.0, several studies on uncertainty quantification of reactor neutronic parameters with JENDL-4.0 have been conducted for various types of fission reactor.[6, 7, 8, 9, 10]

In the present study, we quantify uncertaintis of reactor neutronic parameters of light water reactor fuel cells. In addition to static reactor neutronic parameters such as critical eigenvalues, we consider burnup-related parameters such as burnup loss reactivity and coolant density-reduced reactivity at the end of cycle (EOC). Uncertainty quantification of burnup-related reactor neutronic parameters of light water reactors with the JENDL4.0 covariance data has been already conducted by Sakai et al. with the random sampling technique[8]. In the present study, we perform uncertainty propagation calculations with sensitivity coefficients obtained by the adjoint-based technique: the generalized perturbation theory. Sensitivity coefficients are quite useful quantities to deeply understand the fission chain process and nuclide depletion process occuring in nuclear fission reactors.

We treat several fuel cells composed of different kinds of fissile fuel, such as an uraniumoxide fuel, an uranium-plutonium mixed-oxide fuel, a thorium-uranium mixed-oxide fuel and a thorium-plutonium mixed-oxide fuel. The thorium fuel cells are considered since the thorium fuel has recently received attention of some nuclear engineers in Japan.[11]

As well as the uncertainty of each reactor neutronic parameter, cross-correlations among different reactor neutronic parameters are also important as pointed out by 
Yamamoto.[12] Thus we evaluate the cross-correlations among different reactor neutronic parameters for each fuel cell.

The present paper is organized as follows. Section 2 briefly explains theoretical backgrounds of uncertainty quantification and sensitivity calculation. The generalized perturbation theory employed to calculate burnup-related parameter sensitivities is also described. Section 3 gives information of the numerical calculation, such as specifications of treated fuel cells, the JENDL-4.0 covariance data and employed numerical tools. Numerical results including verification test of burnup-related parameter sensitivity calculations are described in Sec. 4, and all these are summarized in Sec. 5.

\section{Methodology of Uncertainty Quantification}

In the present study, we conduct uncertainty propagation calculations from nuclear data to reactor neutronic parameters. Methods of the uncertainty quantification can be categorized into two groups; the random sampling approach and the sensitivity-based approach.

In the random sampling approach, the covariance matrix of nuclear data is diagonalized, and eigenvalues and eigenvectors of the covariance matrix are obtained. Since there are no cross-correlations among nuclear data parameters in the eigenvector space, a random value is sampled from the Gaussian distributions for each nuclear data in the eigenvector space according to the eigenvalues. Then the obtained nuclear data parameters in the eigenvector space are projected to the original space, and we obtain a nuclear data set in which values are randomly-distrubuted according to the covariance matrix. Using various sets of distributed nuclear data, we can obtain statics of the observed re- 
actor neutronic parameters and quantify their uncertainties. Detail of this procedure is well described in a paper written by Kawano et al.[13] Recently, this approach has been widely adopted in uncertainty quantification by virtue of a drastic increase of computer powers.[14]

The sensitivity based-approach is classical and has been widely utilized in the reactor physics field. Once sensitivity coefficients of reactor neutronic parameters to nuclear data are obtained, uncertainty of the reactor neutronic parameters induced by the nuclear data uncertainty can be easily calculated using the simple uncertainty propagation equation. Moreover, component-wise uncertainties such as nuclide-, reaction- and energy range-wise uncertainties, which are quite beneficial information for experts of nuclear data experiments or evaluations, can be easily evaluated. While sensitivity coefficients can be calculated by the numerical differentiation (called as the forward approach), this requires a large amount of computer resources. On the other hand, the adjoint approach has been establised in the very early day of the reactor physics to overcome this difficulty. In this approach, sensitivity coefficients of arbitrary reactor neutronic parameters including burnup-related ones can be calculated by the generalized perturbation theory. Computational burden of the adjoint approach is usually much smaller than that of the forward approach. In the present section, we breifly describe the methodology of the uncertainty propagation calculation and the theory to calculate sensitivity coefficients of burnuprelated parameters. When the number of observed reactor neutronic parameters become huge, the advantage of the adjoint-based approach over the forward-based approach is lost. In such a case, more sophisticated methodologies have recently been proposed.[15] 


\subsection{Uncertainty Propagation Calculation with Sensitivity Coefficients}

Here we consider a reactor neutronic parameter $R_{i}(i=1,2, \ldots, I)$ and nuclear data $\sigma_{j}(j=1,2, \ldots, J)$. A sensitivity coefficient of $R_{i}$ to $\sigma_{j}, S_{j}^{i}$, is generally defined as

$$
S_{j}^{i}=\frac{\partial R_{i}}{\partial \sigma_{j}} \cdot \frac{\sigma_{j}}{R_{i}}
$$

For simplicity, we write sensitivity coefficients in a matrix form as

$$
\boldsymbol{S}=\left(\begin{array}{cccc}
S_{1}^{1} & S_{1}^{2} & \cdots & S_{1}^{I} \\
S_{2}^{1} & S_{2}^{2} & \cdots & S_{2}^{I} \\
\vdots & \vdots & \ddots & \vdots \\
S_{J}^{1} & S_{J}^{2} & \cdots & S_{J}^{I}
\end{array}\right) .
$$

When we write covariance matrix of the nuclear data as $\boldsymbol{V}_{\sigma}$ which is a $J \times J$ square matrix, an uncertainty propagation equation can be written as

$$
\boldsymbol{V}_{R}=\boldsymbol{S}^{T} \boldsymbol{V}_{\sigma} \boldsymbol{S}
$$

where $\boldsymbol{V}_{R}$ is a covariance matrix of the reactor neutronic parameters, which is a $I \times I$ square matrix, and the superscript $T$ denotes a transpose of matrix.

\subsection{Sensitivity Calculation of Burnup-Related Neutronic Parameters}

Sensitivity coefficients of critical eigenvalue $k_{\text {eff }}$ are easily calculated from forward and adjoint neutron fluxes based on the simple perturbation theory. To obtain sensitivity coefficients of arbitrary static parameters, such as a reaction rate ratio, the generalized perturbation theory (GPT) has been developed and widely utilized.[16] In GPT, sensitivity coefficients are calculated from forward/adjoint and generalized forward/adjoint fluxes. Furthremore, GPT has been extended to calculate sensitivity coefficients of burnup-related 
parameters in a neutron/nuclide coupled field.[17, 18] Since a procedure of sensitivity calculations based on the adjoint-approach is well summarized in various texts and papers, we here describe a procedure to calculate sensitivity coefficients of burnup-related parameters briefly.

First we consider a sensitivity coefficient of number density of nuclide $k$ at $\operatorname{EOC}\left(t_{E}\right)$, $N^{k}\left(t_{E}\right)$, to nuclear data $\sigma$. This sensitivity is defined as

$$
S_{\sigma}^{N^{k}}=\frac{\sigma}{N^{k}\left(t_{E}\right)} \frac{d N^{k}\left(t_{E}\right)}{d \sigma}=\frac{\sigma}{N^{k}\left(t_{E}\right)} \mathbf{e}_{k}^{T} \frac{d \mathbf{N}\left(t_{E}\right)}{d \sigma}
$$

where $\mathbf{e}_{k}$ is a vector in which the $k$ th element is unity and other elements are zero, and $\mathbf{N}\left(t_{E}\right)$ denotes a number density vector at EOC. In order to calculate the derivative in Equation (4), we consider the following burnup equation:

$$
\frac{\partial \mathbf{N}(t)}{\partial t}=\mathbf{M}(t) \mathbf{N}(t)
$$

where $\mathbf{M}$ is a transmutation matrix. By multiplying $\mathbf{w}^{T}(t)$ to the both sides of the above equation and integrating it over $\left[0, t_{E}\right]$, we derive

$$
\mathbf{w}^{T}\left(t_{E}\right) \mathbf{N}\left(t_{E}\right)=\mathbf{w}^{T}(0) \mathbf{N}(0)+\int \frac{\partial \mathbf{w}^{T}}{\partial t} \mathbf{N} d t+\int \mathbf{w}^{T} \mathbf{M} \mathbf{N} d t
$$

Differentiating both sides of Equation (6) by $\sigma$, the following equation is derived:

$$
\frac{d}{d \sigma}\left(\mathbf{w}^{T}\left(t_{E}\right) \mathbf{N}\left(t_{E}\right)\right)=\int \frac{d \mathbf{N}^{T}}{d \sigma}\left\{\frac{\partial \mathbf{w}}{\partial t}+\mathbf{M}^{T} \mathbf{w}\right\} d t+\int \mathbf{w}^{T} \frac{d \mathbf{M}}{d \sigma} \mathbf{N} d t
$$

By setting $\mathbf{w}\left(t_{E}\right)=\mathbf{e}_{k}$, the derivative $\frac{d N^{k}\left(t_{E}\right)}{d \sigma}$ is defined as

$$
\frac{d N^{k}\left(t_{E}\right)}{d \sigma}=\int \frac{d \mathbf{N}^{T}}{d \sigma}\left\{\frac{\partial \mathbf{w}}{\partial t}+\mathbf{M}^{T} \mathbf{w}\right\} d t+\int \mathbf{w}^{T} \frac{d \mathbf{M}}{d \sigma} \mathbf{N} d t
$$

The first integral of the right hand side (RHS) of Equation (8) can be removed by appropriately choosing the weight function $\mathbf{w}$ with $\mathbf{w}\left(t_{E}\right)=\mathbf{e}_{k}$. The choice of the weight function and detail expression of the second term of the RHS can be found elsewhere.[17, 18] 
Next we describe a procedure to calculate a sensitivity coefficient of an arbitrary reactor neutronic parameter at EOC, $R_{E}$. Derivative of $R_{E}$ to nuclear data $\sigma$ can be written as

$$
\frac{d R_{E}}{d \sigma}=\frac{\partial R_{E}}{\partial \sigma}+\left(\frac{\partial R_{E}}{\partial \boldsymbol{\phi}_{E}}\right)^{T}\left(\frac{d \boldsymbol{\phi}_{E}}{d \sigma}\right)+\left(\frac{\partial R_{E}}{\partial \boldsymbol{N}\left(t_{E}\right)}\right)^{T}\left(\frac{d \boldsymbol{N}\left(t_{E}\right)}{d \sigma}\right)
$$

where $\phi_{E}$ is neutron flux distribution at EOC. The first and second terms of RHS in Equation (9) are refered to as a "static term" in the present paper and are easily calculated by GPT. The third term is refered to as a "burnup term" and is calculated as follows. This burnup term is approximated as

$$
\begin{array}{r}
\left(\frac{\partial R_{E}}{\partial \boldsymbol{N}\left(t_{E}\right)}\right)^{T}\left(\frac{d \boldsymbol{N}\left(t_{E}\right)}{d \sigma}\right)=\sum_{k}\left(\frac{\partial R_{E}}{\partial N^{k}\left(t_{E}\right)}\right)\left(\frac{d N^{k}\left(t_{E}\right)}{d \sigma}\right) \\
\approx \frac{d}{d \sigma}\left\{\sum_{k}\left(\frac{\partial R_{E}}{\partial N^{k}\left(t_{E}\right)}\right) N^{k}\left(t_{E}\right)\right\}=\frac{d}{d \sigma}\left\{\left(\frac{\partial R_{E}}{\partial \boldsymbol{N}\left(t_{E}\right)}\right)^{T} \boldsymbol{N}\left(t_{E}\right)\right\} .
\end{array}
$$

We can easily find that this burnup term can be calculated from Equation (7) with $\mathbf{w}\left(t_{E}\right)=\frac{\partial R_{E}}{\partial \boldsymbol{N}\left(t_{E}\right)}$. The final condition of $w\left(t_{E}\right)$, i.e., the derivative of $R_{E}$ to the number density at EOC, is easily calculated by the static GPT. Generally $\boldsymbol{N}\left(t_{E}\right)$ has a large sensitivity to reaction cross sections that are related to nuclide transmutation: capture and fission cross sections. In the present study, therefore, we consider these two reaction types of cross section in calculating $\frac{\partial \boldsymbol{N}\left(t_{E}\right)}{\partial \sigma}$. Note that fission cross sections are important also from a view point of a so called power normalization effect, that is a perturbation in neutron flux level induced by a perturbation in fission cross sections under a condition of fixed power rating.

To calculate sensivitity of reactivity, there are two approaches; one is based on reactivity expression by the perturbation theory and the other is by the two eigenvalues. In the present study, we obtain sensitivity of reactivity from two sensitivities of critical 
eigenvalues as

$$
\frac{d \rho}{d \sigma}=\left(-\frac{1}{k_{1}^{2}} \frac{\partial k_{1}}{\partial \sigma}+\frac{1}{k_{2}^{2}} \frac{\partial k_{2}}{\partial \sigma}\right)
$$

where reactivity $\rho$ is defined from two critical eigenvalues $k_{1}$ and $k_{2}$ as

$$
\rho=\frac{1}{k_{1}}-\frac{1}{k_{2}}
$$

\section{Numerical Condition}

\subsection{Specifications of Fuel Cells}

The present study treats four light water reactor fuel cells composed of different combinations of fissile/fertile materials. A uranium-oxide fuel (U) cell, a uranium-plutonium mixed-oxide fuel (U-Pu) cell and a thorium-uranium mixed-oxide fuel (Th-U) cell are made to represent a $17 \times 17$ pressurized water reactor fuel assembly[19, 20]. Uranium-235 is only a fissile material and no uranium-233 is included in the Th- $\mathrm{U}$ cell. Uranium-235 concentrations in total uranium are $4.1 \mathrm{wt} \%$ for the $\mathrm{U}$ cell, $0.2 \mathrm{wt} \%$ for the $\mathrm{U}-\mathrm{Pu}$ cell and $19.5 \mathrm{wt} \%$ for the Th-U cell, respectively. Plutonium content of the U-Pu cell is $10.0 \mathrm{wt} \%$ and uranium content of the Th-U cell is $25 \mathrm{wt} \%$. Fuel cell specification of a thorium-plutonium mixed oxide fuel (Th-Pu) cell is taken from the IAEA pincell benchmark.[21] The fuel composition of the Th-Pu cell includes $95.5 \mathrm{wt} \%$ of thorium and $4.5 \mathrm{wt} \%$ of reactor-grade plutonium. Fuel cell specifications and burnup conditions for these four fuel cells are summarized in Table 1. Plutonium and americium isotopic compositions of the U-Pu and Th-Pu cells are $(\mathrm{Pu}-238 /-239 /-240 /-241 /-242 / \mathrm{Am}-241)=(0.02 / 0.55 / 0.25 / 0.09 / 0.06 / 0.03)$ and $(0.01 / 0.62 / 0.24 / 0.08 / 0.05 / 0.00)$, respectively. Since moderator-to-fuel volume ratio of the Th-Pu cell is larger than the others, neutron energy spectrum of the Th-Pu cell is 
the softest among four.

\subsection{Covariance Data}

We use all the covariance data of neutron reaction cross sections given to heavy nuclides in JENDL-4.0. Since the original covariance data of uranium-233, -235, -238 and plutonium-239 have problems in their evaluation process, we use the revised data given in the JENDL-4.0 updated files. All the covariance data are processed by the ERRORJ code[22] incorporated into the NJOY code system[23], and the covariance data in a 107group structure are obtained. Incident neutron energy is set to thermal neutron energy in processing of the fission spectrum covariance data.

\subsection{Calculation Tools and Evaluated Neutronic Parameters}

All the numerical calculations are performed by a general-purpose reactor physics code system CBZ, which is being developed at Hokkaido university. Resonance selfshielded cross sections are generated with the equivalence theory using a multi-group library named CBZLIB. Cell heterogeneity is considered by the one-term rational approximation with energy group-wise optimized Bell factors and the Dancoff factor method. The resonance interference effect among heavy nuclides is considered by the multiple Rparameter method[24]. In the present study, a 107-group library based on JENDL-4.0 is utilized.

With the obtained 107-group cross sections, eigenvalue calculations are performed with the collision probability method. In sensitivity calculations, white boundary conditions are assigned to reduce a computational cost. The fuel region and the moderator region 
are both divided into three spatial meshes. Thus, the total number of spatial meshes are seven. Depletion calculations are carried out with the Krylov subspace method.[25] A burnup chain which consists of 193 fission product nuclides is taken from the SRAC code system[26]. Validity of CBZ has been confirmed through an experimental analysis against post-irradiation examination data.[27]

Neutronic parameters of which uncertainties are evaluated are an critical eigenvalue at $\mathrm{BOC}\left(k_{\mathrm{eff}, B O C}\right)$, a burnup reactivity loss $(\mathrm{BRL})$, a coolant density-reduced reactivity $(\mathrm{CDR})$ and an instantaneous conversion ratio (ICR). A coolant density-reduced reactivity is defined as a reactivity caused by a 10\% decrease in hydrogen and oxigen number densities in a moderator region. An instantaneous conversion ratio is defined as a ratio of macroscopic capture reaction rate of fertile nuclides (thorium-232, uranium-238 and plutonium-240) to macroscopic absorption reaction rate of fissile nuclides (uranium-233, -235 and plutonium-239 and -241).

\section{Numerical Result}

Table 2 shows calculated neutronic parameters of four types of light water reactor fuel cells. Generally speaking, a burnup reactivity loss becomes small when a conversion ratio takes a large value. It is interesting to point out that the burnup reactivity loss normalized by burnup of the Th-Pu cell is larger than those of the U-Pu and Th-U cells even though the instantaneous conversion ratios at BOC and EOC of the Th-Pu cell are comparable to or larger than those of the U-Pu and Th-U cells. This could be understood from a difference in a neutron production cross section $\left(\nu \sigma_{f}\right)$ of each fissile material. Figure 1 shows burnup-dependent 1-group production cross sections of uranium-233, -235 
and plutonium-239 calculated in the Th-U cell. In the Th-Pu cell, plutonium-239, which has a large production cross section, is depleted and uranium-233, which has a relatively small production cross section, is produced via neutron capture reaction of thorium-232. Thus, a ‘conversion' from plutonium-239 to uranium-233 gives negative impact on neutron multiplication in comparison with a 'conversion' from plutonium-239 to plutonium-239 in the U-Pu cell and uranium-235 to plutonium-239 in the Th- $\mathrm{U}$ cell. The Th-Pu cell also has its own specific feature of burnup dependence of coolant density-reduced reactivity: coolant density-reduced reactivity at EOC is smaller than that at BOC. This is because most of plutonium isotopes charged at BOC are depleted through fuel burnup and neutron energy spectrum at EOC becomes much softer than that at BOC as shown in Fig. 2.

\subsection{Verification of Sensitivity of Burnup-Related Parameters}

In order to verify a GPT-based sensitivity calculation capability of CBZ, sensitivities calculated with GPT are compared with reference sensitivities, which are obtained by numerical differentiation in which a relative perturbation of $1 \%$ is given to a specific nuclear data parameter and change in a response neutronic parameter is observed. Comparisons are made for sensitivities of a critical eigenvalue and an instantaneous conversion ratio at EOC of the $\mathrm{U}$ cell to uranium-235 fission and uranium-238 capture cross sections. Results are shown in Figs. 3 to 6 . Sensitivities without the burup term are also shown to see the burnup effect on neutronic parameters at EOC. Sensitivities calculated with GPT agree well with the references and the GPT-based sensitivity calculation capability of CBZ is well verified. A positive effect of the burnup term observed in a sensitivity of critical eigenvalue to uranium-238 capture cross section can be easily understood in terms 
of a conversion of uranium-238 to plutonium-239. Negative effect of the burnup term is observed in a sensitivity of critical eigenvalue to uranium-235 fission cross section. The reason of this negative effect is explained from the power normalization effect; When a uranium-235 fission cross section is slightly increased, neutron flux level decreases because of the fixed power rating and conversion of uranium-238 to plutonium-239 would be restricted. As a result, a critical eigenvalue becomes small.

\subsection{Uncertainty Quantification of Neutronic Parameters}

Uncertainties of neutronic parameters of four fuel cells are quantified using sensitivity coefficients calculated with GPT and the JENDL-4.0 covariance data in a multi-group form. Relative standard deviations of neutronic parameters are summarized in Table 3 and component-wise contributions are shown in Table 4 for the $\mathrm{U}$ cell and Table 5 for the U-Pu cell.

In the U cell, uncertainty of uranium-238 capture cross section is dominant in all the neutronic parameters. Uncertainties of $\nu$ value (a number of neutrons generated by a fission reaction) of uranium-235 and $\chi$ (fission spectrum) of fissile nuclides are also dominant components. Uranium-238-induced uncertainty of instantaneous conversion ratio at EOC is smaller than that at BOC due to cancellation in sensitivities of instantaneous conversion ratio to uranium-238 capture cross section between the static term and the burnup term as showin in Fig. 5.

In the U-Pu cell, it is a bit surprising to see that uncertainties induced by capture cross sections of high plutonium isotopes, plutonium-240 and -241, are dominent components in several neutronic parameters. Figure 7 shows relative standard deviations of capture 
cross sections of uranium-238 and plutonium-239,-240 and -241 in a 107-group structure.

We can find that uncertainties of capture cross sections of plutonium-240 and -241 are much larger than the others. It should be noted that strong positive correlations are also given in capture cross sections of plutonium-240 below $8 \mathrm{eV}$ and plutonium-241 below $350 \mathrm{eV}$, respectively, in the covariance matrix. Uncertainty of coolant densityreduced reactivity induced by $\chi$ of plutonium- 241 is larger than that by $\chi$ of plutonium-239 since the plutonium composition changes through burnup; The plutonium composition of BOC is $(\mathrm{Pu}-238 /-239 /-240 /-241 /-242)=(0.02 / 0.56 / 0.26 / 0.10 / 0.07)$ and that at $\mathrm{EOC}$ is $(0.04 / 0.41 / 0.29 / 0.16 / 0.09)$

In the Th- $\mathrm{U}$ and $\mathrm{Th}-\mathrm{Pu}$ cells, uncertainty of capture cross section of thorium-232 is a main contributor to uncertainties of all the neutronic parameters. Table 6 shows energy range-wise neutronic parameter uncertainties of the Th-Pu cell. We can find that the thorium-232 capture cross section uncertainty in the intermediate energy range from $78.9 \mathrm{eV}$ to $86.5 \mathrm{keV}$ is dominant in the neutronic parameter uncertainties and that in the thermal energy range is relatively small. It has been reported that the thorium-232 capture cross section uncertainty given in JENDL-4.0 is significantly larger than that given in ENDF/B-VII.1 even though the same resonance parameter set is adopted to both the evaluated data.[10]

Tables 7 to 10 show correlation among different neutronic parameters for each fuel cell. A strong correlations are observed between the same neutronic parameters at BOC and EOC. It suggests that uncertainties of neutronic parameters at EOC that are not measurable can be reduced by some techniques if the parameter at BOC is measurable through reactor physics tests conducted in start-up process. Furthremore, even among the different kinds of neutronic parameters, relatively strong correlations are observed since 
the uncertainties of the same nuclear data, such as capture cross sections of uranium-238 and thorium-232, contribute to uncertainties of different neutronic parameters. Note that correlation among neutronic parameters in the $\mathrm{U}-\mathrm{Pu}$ cell is relatively weak in comparison with those in the other cells. That is because main contributors to neutronic parameter uncertainties are slightly different among neutronic parameters in the U-PU cell as shown in Table 5. A weak correlation between the instantaneous conversion ratio at EOC and the coolant density-reduced reactivities at BOC and EOC in the Th-Pu cell is also notable. That comes from a difference in energy profile of sensitivity coefficients as shown in Fig. 8

\section{Conclusion}

Neutronic parameter uncertainty induced by nuclear data uncertainty has been successfully quantified for several light water reactor fuel cells composed of different combinations of fissile/fertile nuclides. The covariance data given in JENDL-4.0 has been used as the nuclear data uncertainty and uncertainty propagation calculations have been carried out using sensitivity coefficients calculated with the generalized perturbation theory for burnup-related neutronic parameters. The calculated sensitivity coefficients have been verified through a comparison with those obtained with the numerical differetiation.

We have found that main contributors of nuclear data uncertainty to the neutronic parameter uncertainty are uranium-238 capture cross section in the $\mathrm{U}$ cell, plutonium240 and -241 capture cross sections and fission spectrum of fissile plutonium isotopes in the U-Pu cell. We have also found that thorium-232 capture cross section uncertainty is a dominant source of neutronic parameter uncertainty in the Th- $\mathrm{U}$ and Th-Pu cells and that the parameter uncertainties in the $\mathrm{Th}-\mathrm{U}$ and $\mathrm{Th}-\mathrm{Pu}$ cells are much larger than those of the 
other two. These quantitative uncertainty information can be a driven force for nuclear data experts to begin new measurements/evaluations. It should be emphasized that precise and detail information of component-wise uncertainties can be obtained by virtue of the adjoint-based sensitivity calculation methodology. Furthermore, cross-correlations have also been evaluated for each fuel cell, and strong correlations among the same parameters at BOC and EOC and among different parameters have been observed. This result is quite optimistic to introduce the uncertainty reduction technique, such as one proposed by Yamamoto[12], for unmeasurable parameters using other measurable parameters.

\section{Acknowledgment}

This work was supported by JSPS KAKENHI Grant Number 24561040.

\section{References}

1) M. B. Chadwick, M. Herman, P. Oblozinský, M. E. Dunn, Y. Danon, A. C. Kahler, D. L. Smith, B. Pritychenko, G. Arbanas, R. Arcilla, R. Brewer, D. A. Brown, R. Capote, A. D. Carlson, Y. S. Cho, H. Derrien, K. Guber, G. M. Hale, S. Hoblit, S. Holloway, T. D. Johnson, T. Kawano, B. C. Kiedrowski, H. Kim, S. Kunieda, N. M. Larson, L. Leal, J. P. Lestone, R. C. Little, E. A. McCutchan, R. E. MacFarlane, M. MacInnes, C. M. Mattoon, R. D. McKnight, S. F. Mughabghab, G. P. A. Nobre, G. Palmiotti, A. Palumbo, M. T. Pigni, V. G. Pronyaev, R. O. Sayer, A. A. Sonzogni, N. C. Summers, P. Talou, I. J. Thompson, A. Trkov, R. L. Vogt, S. C. van der Marck, A. Wallner, M. C. White, D. Wiarda, P. G. Young, ENDF/B-VII.1 nuclear data for science and technology: cross sections, covariances, fission product yields and decay data, Nucl. Data Sheets, 112(2011), pp. 2887-2996. 
2) K. Shibata, T. Kawano, T. Nakagawa, O. Iwamoto, J. Katakura, T. Fukahori, S. Chiba, A. Hasegawa, T. Murata, H. Matsunobu, T. Ohsawa, Y. Nakajima, T. Yoshida, A. Zukeran, M. Kawai, M. Baba, M. Ishikawa, T. Asami, T. Watanabe, Y. Watanabe, M. Igashira, N. Yamamuro, H. Kitazawa, N. Yamano, H. Takano, Japanese evaluated nuclear data library version 3 revision-3: JENDL-3.3, J. Nucl. Sci. Technol., 39[11](2002), pp. 1125-1136.

3) M. Ishikawa, Recent application of nuclear data to fast reactor core analysis and design in Japan, Proc. Inf. Conf. on Nucl. Data for Sci. and Technol. (ND2004), Santa Fe, New Mexico, Sep. 26-Oct. 1. Am. Inst. of Phys., 1405, (2005).

4) T. Sugawara, K. Nishihara, K. Tsujimoto, T. Sasa, H. Oigawa, Analytical validation of uncertainty in reactor physics parameters for nuclear transmutation systems, J. Nucl. Sci. Technol., 47[6](2010), pp. 521-530.

5) K. Shibata, O. Iwamoto, T. Nakagawa, N. Iwamoto, A. Ichihara, S. Kunieda, S. Chiba, K. Furutaka, N. Otuka, T. Ohsawa, T. Murata, H. Matsunobu, A. Zukeran, S. Kamada, J. Katakura, JENDL-4.0: a new library for nuclear science and engineering, J. Nucl. Sci. Technol., 48[1](2011), pp. 1-30.

6) K. Sugino, M. Ishikawa, K. Yokoyama, Y. Nagaya, G. Chiba, T. Hazama, T. Kugo, Development of a unified cross-section set ADJ2010 based on adjustment technique for fast reactor core design, $J$. Korean Phys. Soc., 59[2](2011), pp. 1357-1360.

7) I. Kodeli, L. Snoj, Evaluation and uncertainty analysis of the KRITZ-2 critical benchmark experiments, Nucl. Sci. Eng.,171(2012), pp. 231-238.

8) T. Sakai, S. Shiba, K. Saitou, Analysis of UAM benchmark problems [6] uncertainty analysis of core problems using stochastic sampling method, Proc. of the 2012 fall meeting of the Atomic Energy Society of Japan,(2012),[in Japanese][CD-ROM].

9) A. Oizumi, K. Yokoyama, M. Ishikawa, T. Kugo, Uncertainty evaluation for MA production in spent light water reactor fuel with use of burnup sensitivity analysis, Proc. of the 2012 Annual Symposium 

on Nuclear Data (NDS 2012), Kyoto, Japan, Nov. 15-16, 2012 (2012), [to be publised].

10) T. Kojima, T. Fujii, T. Kitada, The validity of covariance data of ${ }^{232}$ Th in JENDL-4.0, ENDF/BVII.1 and ENDF/Bi-VI.8, Proc. of the 2012 Annual Symposium on Nuclear Data (NDS 2012), Kyoto, Japan, Nov. 15-16, 2012 (2012), [to be publised].

11) S. Permana, N. Takaki, H. Sekimoto, Feasible region of design parameters for water cooled thorium breeder reactor, J. Nucl. Sci. Technol., 44[7](2007), pp. 946-957.

12) A. Yamamoto, Y. Yasue, T. Endo, Y. Kodama, Y. Ohoka, M. Tatsumi, Uncertainty estimation of core safety parameters using cross-correlations of covariance matrix, Proc. Int. Conf. on Physics of Reactors (PHYSOR2012), Knoxville, Tennessee, April 15-20, 2012 (2012),[CD-ROM].

13) T. Kawano, K. M. Hanson, S. Frankle, P. Talou, M. B. Chadwick, R. C. Little, Evaluation and propagation of the ${ }^{239} \mathrm{Pu}$ fission cross-section uncertainties using a Monte Carlo technique, Nucl. Sci. Eng., 153(2006), pp. 1-7.

14) D. Rochman, A. J. Koning, S. C. van der Marck, Uncertainties for criticality-safety benchmarks and $k_{\text {eff }}$ distributions, Ann. Nucl. Energy, 36(2009), pp. 810-831.

15) H. S. Abdel-Khalik, P. J. Turinsky, M. A. Jessee, Efficient subspace methods-based algorithms for performing sensitivity, uncertainty, and adaptive simulation of large-scale computational models, Nucl. Sci. Eng., 159(2008), pp. 256-272.

16) M.L.Williams, 'Perturbation theory for reactor analysis,' CRC Handbook of Nuclear Reactors Calculations, Vol.3, pp.63, CRC Press, Boca Raton, Florida (1986).

17) M.L. Williams, "Development of depletion perturbation theory for coupled neutron/nuclide fields," Nucl. Sci. Eng., 70(1979), pp. 20-36.

18) T. Takeda, T. Umano, "Burnup sensitivity analysis in a fast breeder reactor - Part I: Sensitivity calculation method with generalized perturbation theory," Nucl. Sci. Eng., 91(1985), pp. 1-10.

19) J. Katakura, M. Kataoka, K. Suyama, T. Jin, S. Ohki, A set of ORIGEN2 cross section libraries based 
on JENDL-3.3 library: ORLIBJ33, JAERI-Data/Code 2004-015, Japan Atomic Energy Research Institute (2004).[in Japanese]

20) X. Zhao, E. E. Pilat, K. D. Weaver, P. Hejzlar, A PWR thorium pin cell burnup benchmark, Proc. Int. Conf. on Physics of Reactors (PHYSOR2000), Pittsburgh, Pennsylvania, May 7-11, 2000 (2000),[CD$\mathrm{ROM}]$.

21) Potential of thorium based fuel cycles to constrain plutonium and reduce long lived waste toxicity. IAEA-TECDOC-1319 (2002).

22) G. Chiba, ERRORJ: A code to process neutron-nuclide reaction cross section covariance, version 2.3, JAEA-Data/Code 2007-007, Japan Atomic Energy Agency (2007).

23) R. E. MacFarlane, A. C. Kahler, Methods for processing ENDF/B-VII with NJOY, Nucl. Data Sheets, 111(2010), pp. 2739-2890.

24) G. Chiba, CBGLIB: A multi-group neutron library for precise neutronics simulations, JAEA-Conf 2008-008, Japan Atomic Energy Agency (2008), pp. 114-120.

25) A. Yamamoto, M. Tatsumi, N. Sugimura, Numerical solution of stiff burnup equation with short half lived nuclides by the Krylov subspace method, J. Nucl. Sci. Technol., 44(2007), pp. 147-154.

26) K. Okumura, T. Kugo, K. Kaneko, K. Tsuchihashi, SRAC2006: a comprehensive neutronics calculation code system, JAEA-Data/Code 2007-004, Japan Atomic Energy Agency (2007).

27) Y.Kawamoto, G. Chiba, M. Tsuji, T. Narabayashi, Validation of CBZ code system for post irradiation examination analysis and sensitivity analysis of $(\mathrm{n}, \gamma)$ branching ratio, Proc. of the 2012 Annual Symposium on Nuclear Data (NDS 2012), Kyoto, Japan, Nov. 15-16, 2012 (2012),[to be publised]. 
Table 1 Geometric specifications of fuel cells and burnup conditions

\begin{tabular}{cccc}
\hline Item & $\mathrm{U} / \mathrm{U}-\mathrm{Pu}$ & $\mathrm{Th}-\mathrm{U}$ & $\mathrm{Th}-\mathrm{Pu}$ \\
\hline Pin pitch $[\mathrm{cm}]$ & 1.265 & 1.2626 & 1.507 \\
Fuel pellet radius $[\mathrm{cm}]$ & 0.412 & 0.41274 & 0.47 \\
Outer radius of cladding region $[\mathrm{cm}]$ & 0.476 & 0.47609 & 0.54 \\
$\mathrm{~V}_{m} / \mathrm{V}_{f}$ & 1.67 & 1.65 & 1.95 \\
Linear heat rating $[\mathrm{W} / \mathrm{cm}]$ & 179 & 171 & 211 \\
Burnup $[\mathrm{GWd} / \mathrm{t}]$ & 45 & 72.189 & 60 \\
Fuel temperature $[\mathrm{K}]$ & 968.8 & 900.0 & 1023 \\
& & & \\
Cladding temparature $[\mathrm{K}]$ & 604.0 & 621.1 & 621.1 \\
Coolant temparature $[\mathrm{K}]$ & 574.2 & 583.1 & 583 \\
\hline
\end{tabular}


Table 2 Calculated neutronic parameters

\begin{tabular}{|c|c|c|c|c|}
\hline Item & $\mathrm{U}$ & $\mathrm{U}-\mathrm{Pu}$ & Th-U & $\mathrm{Th}-\mathrm{Pu}$ \\
\hline Bunup $[\mathrm{GWd} / \mathrm{t}]$ & 45 & 45 & 72.189 & 60 \\
\hline$k_{\text {eff }}$ at $\mathrm{BOC}$ & 1.32736 & 1.13894 & 1.24111 & 1.13118 \\
\hline$k_{\text {eff }}$ at EOC & 0.91262 & 0.98372 & 0.88094 & 0.83392 \\
\hline Burnup reactivity loss $\left[\mathrm{dk} / \mathrm{kk}^{\prime}\right]$ & -0.342 & -0.139 & -0.329 & -0.315 \\
\hline Burnup reactivity loss $\left[\left(\mathrm{dk} / \mathrm{kk}^{\prime}\right) /(\mathrm{GWd} / \mathrm{t})\right]$ & -0.0076 & -0.0031 & -0.0046 & -0.0053 \\
\hline Instantaneous conversion ratio at $\mathrm{BOC}$ & 0.413 & 0.631 & 0.542 & 0.614 \\
\hline Instantaneous conversion ratio at EOC & 0.744 & 0.734 & 0.865 & 0.938 \\
\hline Coolant density-reduced reactivity at BOC [\% dk/kk'] & -1.01 & -1.40 & -1.50 & -1.80 \\
\hline Coolant density-reduced reactivity at EOC [\% $\left.\mathrm{dk} / \mathrm{kk}^{\prime}\right]$ & -2.26 & -1.92 & -2.35 & -1.55 \\
\hline
\end{tabular}


Table 3 Relative standard deviations of neutronic parameters (unit:\%)

\begin{tabular}{|c|c|c|c|c|}
\hline & $\mathrm{U}$ & $\mathrm{U}-\mathrm{Pu}$ & Th-U & $\mathrm{Th}-\mathrm{Pu}$ \\
\hline$k_{\text {eff }}$ at $\mathrm{BOC}$ & 0.538 & 0.888 & 2.786 & 2.689 \\
\hline Burnup reactivity loss & 1.11 & 4.95 & 10.49 & 14.53 \\
\hline Instantaneous conversion ratio at $\mathrm{BOC}$ & 1.53 & 2.04 & 8.16 & 7.36 \\
\hline Instantaneous conversion ratio at EOC & 0.57 & 0.87 & 0.90 & 1.48 \\
\hline Coolant density-reduced reactivity at BOC & 2.26 & 2.18 & 11.03 & 11.55 \\
\hline Coolant density-reduced reactivity at EOC & 1.77 & 2.22 & 6.33 & 15.71 \\
\hline
\end{tabular}


Table 4 Component-wise neutronic parameter uncertainties of $\mathrm{U}$ cell

\begin{tabular}{|c|c|c|}
\hline & & Relative standard deviation (\%) \\
\hline \multirow[t]{3}{*}{$k_{\text {eff }}$ at $\mathrm{BOC}$} & U-238 capture & 0.391 \\
\hline & $\mathrm{U}-235 \nu$ & 0.271 \\
\hline & U-235 capture & 0.166 \\
\hline \multirow[t]{2}{*}{ Burnup reactivity loss } & U-238 capture & 1.03 \\
\hline & $\mathrm{U}-235 \nu$ & 0.36 \\
\hline Instantaneous conversion ratio at $\mathrm{BOC}$ & U-238 capture & 1.52 \\
\hline Instantaneous conversion ratio at EOC & U-238 capture & 0.50 \\
\hline \multirow[t]{2}{*}{ Coolant density-reduced reactivity at BOC } & U-238 capture & 1.96 \\
\hline & $\mathrm{U}-235 \chi$ & 0.94 \\
\hline \multirow[t]{3}{*}{ Coolant density-reduced reactivity at EOC } & U-238 capture & 1.20 \\
\hline & $\mathrm{Pu}-241 \chi$ & 0.61 \\
\hline & $\mathrm{Pu}-239 \chi$ & 0.59 \\
\hline
\end{tabular}


Table 5 Component-wise neutronic parameter uncertainties of U-Pu cell

\begin{tabular}{|c|c|c|}
\hline & & Relative standard deviation (\%) \\
\hline \multirow[t]{4}{*}{$k_{\text {eff }}$ at $\mathrm{BOC}$} & $\mathrm{Pu}-240$ capture & 0.614 \\
\hline & U-238 capture & 0.271 \\
\hline & $\mathrm{Pu}-241$ capture & 0.261 \\
\hline & $\mathrm{Pu}-239$ fission & 0.248 \\
\hline \multirow[t]{3}{*}{ Burnup reactivity loss } & Pu-240 capture & 3.32 \\
\hline & $\mathrm{Pu}-241$ capture & 3.10 \\
\hline & Am-241 capture & 1.29 \\
\hline Instantaneous conversion ratio at $\mathrm{BOC}$ & Pu-240 capture & 1.83 \\
\hline \multirow[t]{2}{*}{ Instantaneous conversion ratio at EOC } & U-238 capture & 0.60 \\
\hline & $\mathrm{Pu}-240$ capture & 0.57 \\
\hline \multirow[t]{3}{*}{ Coolant density-reduced reactivity at BOC } & U-238 capture & 1.21 \\
\hline & $\mathrm{Pu}-239 \chi$ & 0.94 \\
\hline & $\mathrm{Pu}-241 \chi$ & 0.88 \\
\hline \multirow[t]{3}{*}{ Coolant density-reduced reactivity at EOC } & $\mathrm{Pu}-241 \chi$ & 1.45 \\
\hline & $\mathrm{Pu}-240$ capture & 0.79 \\
\hline & $\mathrm{Pu}-239 \chi$ & 0.76 \\
\hline
\end{tabular}


Table 6 Energy range-wise neutronic parameter uncertainties of Th-Pu cell (unit:\%)

\begin{tabular}{ccccccc}
\hline & $k_{\text {eff }, B O C}$ & BRL & ICR $_{B O C}$ & $\mathrm{ICR}_{E O C}$ & $\mathrm{CDR}_{B O C}$ & $\mathrm{CDR}_{E O C}$ \\
\hline Range 1 - Range 1 & 1.62 & 9.2 & 4.6 & 0.2 & 8.3 & 11.0 \\
Range 2 - Range 2 & 0.95 & 5.3 & 2.7 & 0.1 & 4.0 & 5.2 \\
Range 3 - Range 3 & 0.92 & 4.5 & 2.5 & 1.4 & 0.5 & 3.6 \\
Range 1 - Range 2 & 1.45 & 8.2 & 4.1 & 0.1 & 6.7 & 8.9 \\
Range 2 - Range 3 & 0.20 & 1.0 & 0.5 & 0.0 & 0.8 & 1.0 \\
\hline Range 1: 78.9(eV)-86.5(keV), Range 2: $0.6(\mathrm{eV})-78.9(\mathrm{eV})$, Range 3: $-0.6(\mathrm{eV})$
\end{tabular}


Table 7 Correlation of neutronic parameter uncertainties of $U$ cell

\begin{tabular}{|c|c|c|c|c|c|c|}
\hline & $k_{\mathrm{eff}, B O C}$ & BRL & $\mathrm{ICR}_{B O C}$ & $\mathrm{ICR}_{E O C}$ & $\mathrm{CDR}_{B O C}$ & $\mathrm{CDR}_{E O C}$ \\
\hline$k_{\mathrm{eff}, B O C}$ & & -0.89 & -0.73 & -0.68 & 0.71 & 0.55 \\
\hline BRL & & & 0.92 & 0.82 & -0.76 & -0.57 \\
\hline $\mathrm{ICR}_{B O C}$ & & & & 0.87 & -0.83 & -0.64 \\
\hline $\mathrm{ICR}_{E O C}$ & & & & & -0.74 & -0.69 \\
\hline $\mathrm{CDR}_{B O C}$ & & & & & & 0.73 \\
\hline $\mathrm{CDR}_{E O C}$ & & & & & & \\
\hline
\end{tabular}


Table 8 Correlation of neutronic parameter uncertainties of U-Pu cell

\begin{tabular}{|c|c|c|c|c|c|c|}
\hline & $k_{\mathrm{eff}, B O C}$ & BRL & $\mathrm{ICR}_{B O C}$ & $\mathrm{ICR}_{E O C}$ & $\mathrm{CDR}_{B O C}$ & $\mathrm{CDR}_{E O C}$ \\
\hline$k_{\mathrm{eff}, B O C}$ & & -0.31 & -0.76 & -0.73 & 0.36 & 0.01 \\
\hline BRL & & & 0.72 & 0.62 & -0.12 & 0.43 \\
\hline $\mathrm{ICR}_{B O C}$ & & & & 0.90 & -0.35 & 0.20 \\
\hline $\mathrm{ICR}_{E O C}$ & & & & & -0.47 & 0.04 \\
\hline $\mathrm{CDR}_{B O C}$ & & & & & & 0.78 \\
\hline $\mathrm{CDR}_{E O C}$ & & & & & & \\
\hline
\end{tabular}


Table 9 Correlation of neutronic parameter uncertainties of Th- $\mathrm{U}$ cell

\begin{tabular}{|c|c|c|c|c|c|c|}
\hline & $k_{\mathrm{eff}, B O C}$ & BRL & $\mathrm{ICR}_{B O C}$ & $\mathrm{ICR}_{E O C}$ & $\mathrm{CDR}_{B O C}$ & $\mathrm{CDR}_{E O C}$ \\
\hline$k_{\mathrm{eff}, B O C}$ & & -0.99 & -0.99 & -0.96 & 0.84 & 0.43 \\
\hline BRL & & & 1.00 & 0.97 & -0.88 & -0.51 \\
\hline $\mathrm{ICR}_{B O C}$ & & & & 0.97 & -0.85 & -0.46 \\
\hline $\mathrm{ICR}_{E O C}$ & & & & & -0.85 & -0.50 \\
\hline $\mathrm{CDR}_{B O C}$ & & & & & & 0.84 \\
\hline $\mathrm{CDR}_{E O C}$ & & & & & & \\
\hline
\end{tabular}


Table 10 Correlation of neutronic parameter uncertainties of Th-Pu cell

\begin{tabular}{|c|c|c|c|c|c|c|}
\hline & $k_{\mathrm{eff}, B O C}$ & BRL & $\mathrm{ICR}_{B O C}$ & $\mathrm{ICR}_{E O C}$ & $\mathrm{CDR}_{B O C}$ & $\mathrm{CDR}_{E O C}$ \\
\hline$k_{\mathrm{eff}, B O C}$ & & -0.97 & -0.98 & -0.39 & 0.89 & 0.78 \\
\hline BRL & & & 0.98 & 0.38 & -0.92 & -0.81 \\
\hline $\mathrm{ICR}_{B O C}$ & & & & 0.39 & -0.91 & -0.79 \\
\hline $\mathrm{ICR}_{E O C}$ & & & & & -0.09 & 0.05 \\
\hline $\mathrm{CDR}_{B O C}$ & & & & & & 0.97 \\
\hline $\mathrm{CDR}_{E O C}$ & & & & & & \\
\hline
\end{tabular}




\section{Figure captions}

Figure 1. One-group production cross sections of uranium-233, -235 and plutonium-239 in Th-U cell

Figure 2. Neutron flux energy spectra of Th-Pu cell

Figure 3. Sensitivity of critical eigenvalue at EOC to uranium-238 capture cross section

Figure 4. Sensitivity of critical eigenvalue at EOC to uranium-235 fission cross section

Figure 5. Sensitivity of instantaneous conversion ratio at EOC to uranium-238 capture cross section

Figure 6. Sensitivity of instantaneous conversion ratio at EOC to uranium-235 fission cross section

Figure 7. Relative standard deviations of capture cross sections in JENDL-4.0

Figure 8. Sensitivity of neutronic parameters in Th-Pu cell 


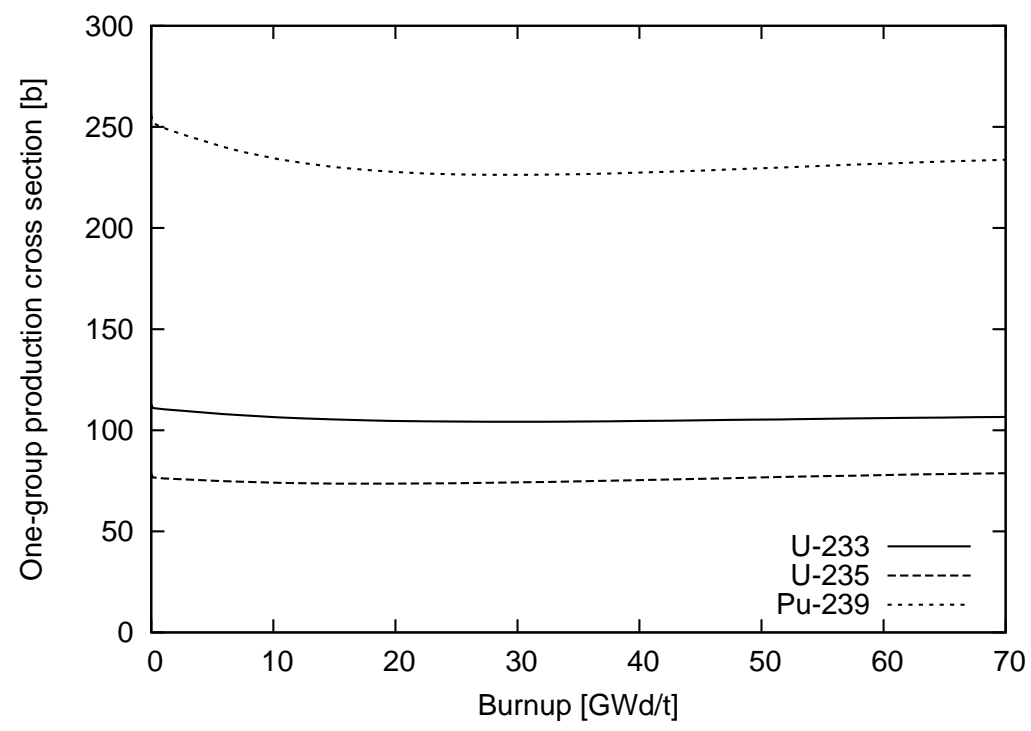

Figure 1 One-group production cross sections of uranium-233, -235 and plutonium-239 in Th-U cell

G.Chiba:

Uncertainty quantification of neutronic parameters of light water reactor fuel cells with JENDL-4.0 covariance data Efficient Fission Neutron Spectrum Matrix Representation by Singular Value Decomposition Technique 


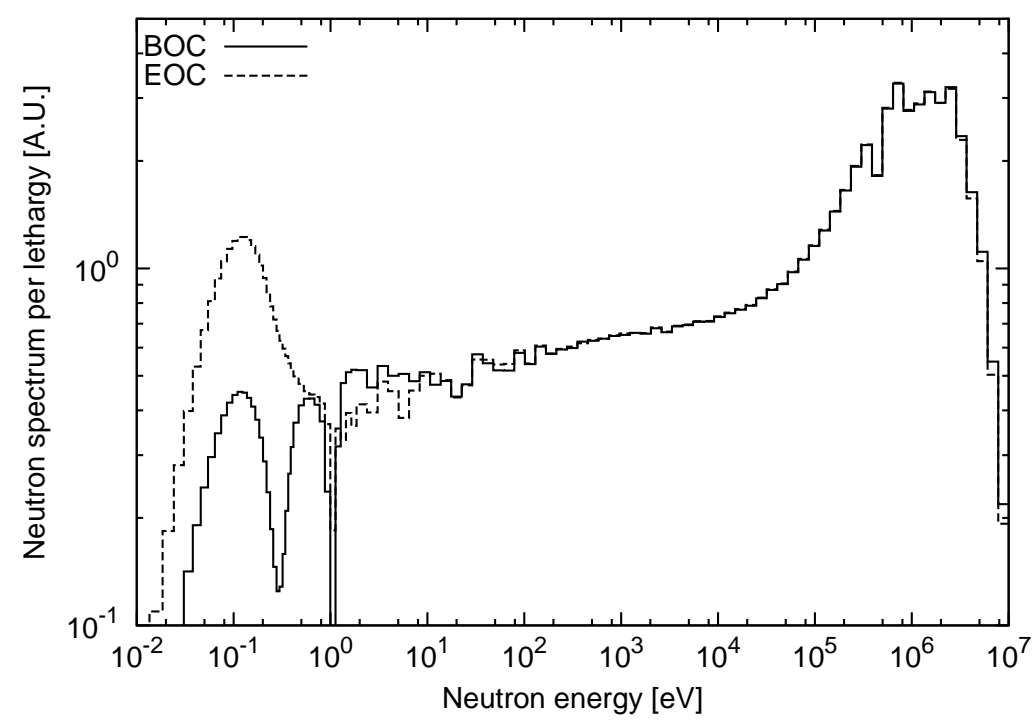

Figure 2 Neutron flux energy spectra of Th-Pu cell

G.Chiba:

Uncertainty quantification of neutronic parameters of light water reactor fuel cells with JENDL-4.0 covariance data 


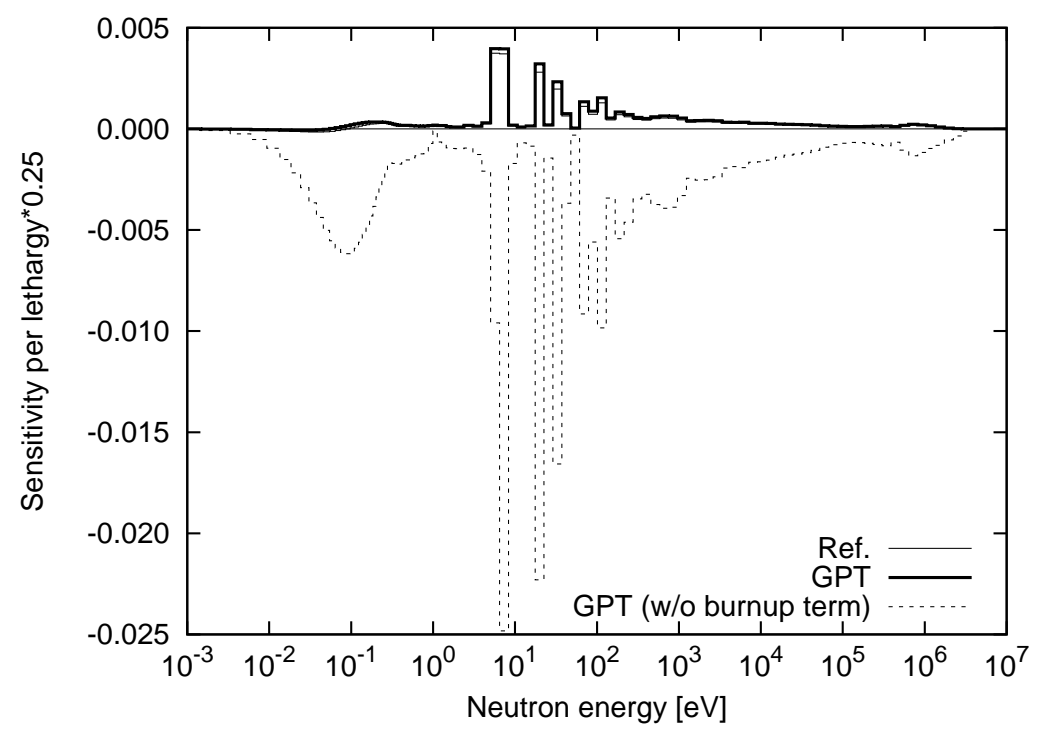

Figure 3 Sensitivity of critical eigenvalue at EOC to uranium-238 capture cross section

\section{G.Chiba:}

Uncertainty quantification of neutronic parameters of light water reactor fuel cells with JENDL-4.0 covariance data 


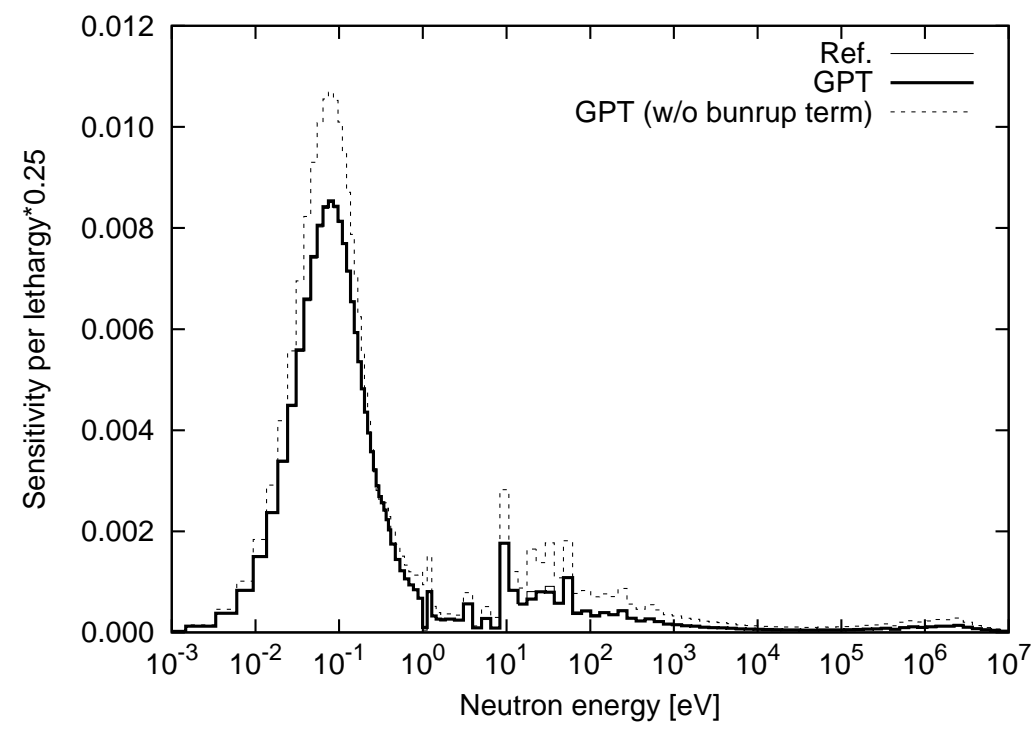

Figure 4 Sensitivity of critical eigenvalue at EOC to uranium-235 fission cross section

\section{G.Chiba:}

Uncertainty quantification of neutronic parameters of light water reactor fuel cells with JENDL-4.0 covariance data 


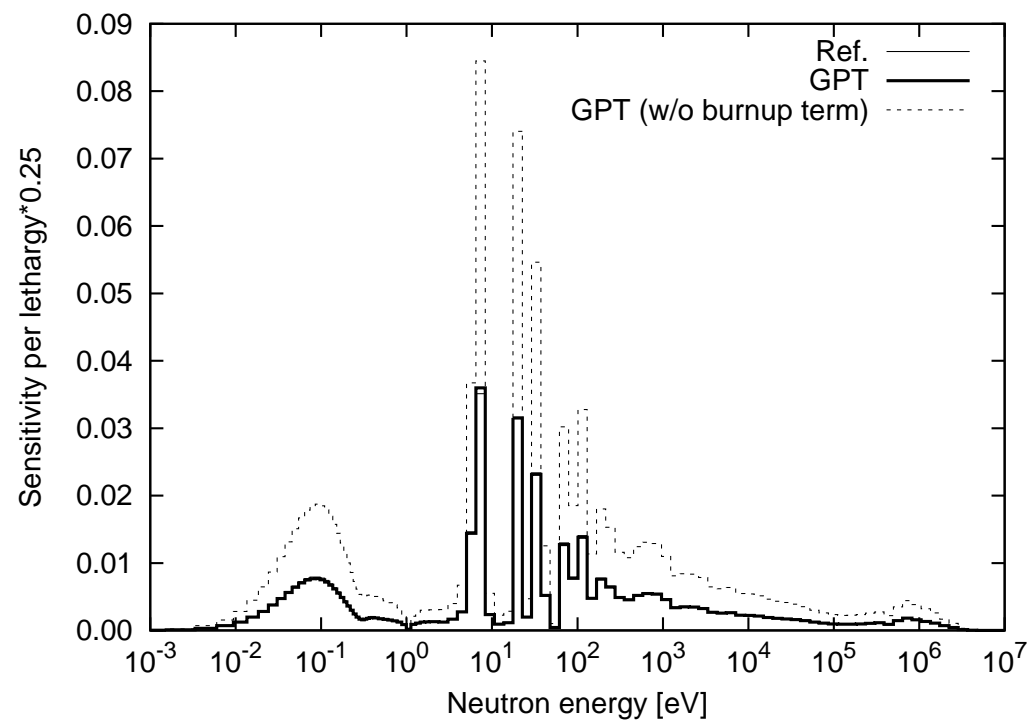

Figure 5 Sensitivity of instantaneous conversion ratio at EOC to uranium-238 capture cross section

G.Chiba:

Uncertainty quantification of neutronic parameters of light water reactor fuel cells with JENDL-4.0 covariance data 


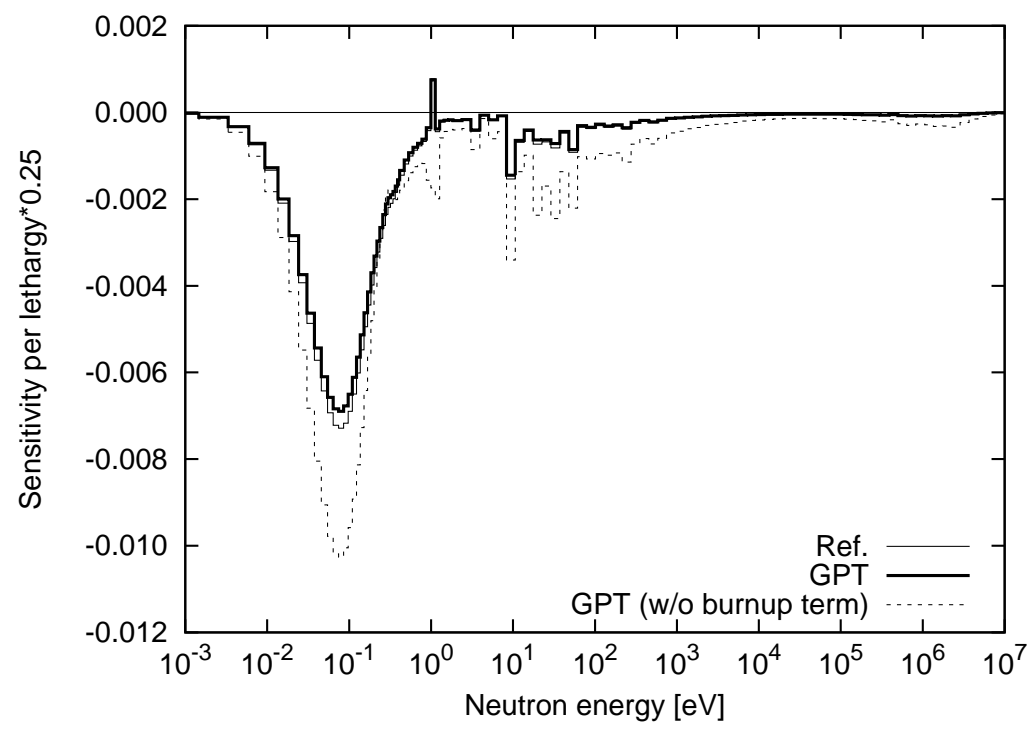

Figure 6 Sensitivity of instantaneous conversion ratio at EOC to uranium-235 fission cross section

\section{G.Chiba:}

Uncertainty quantification of neutronic parameters of light water reactor fuel cells with JENDL-4.0 covariance data 


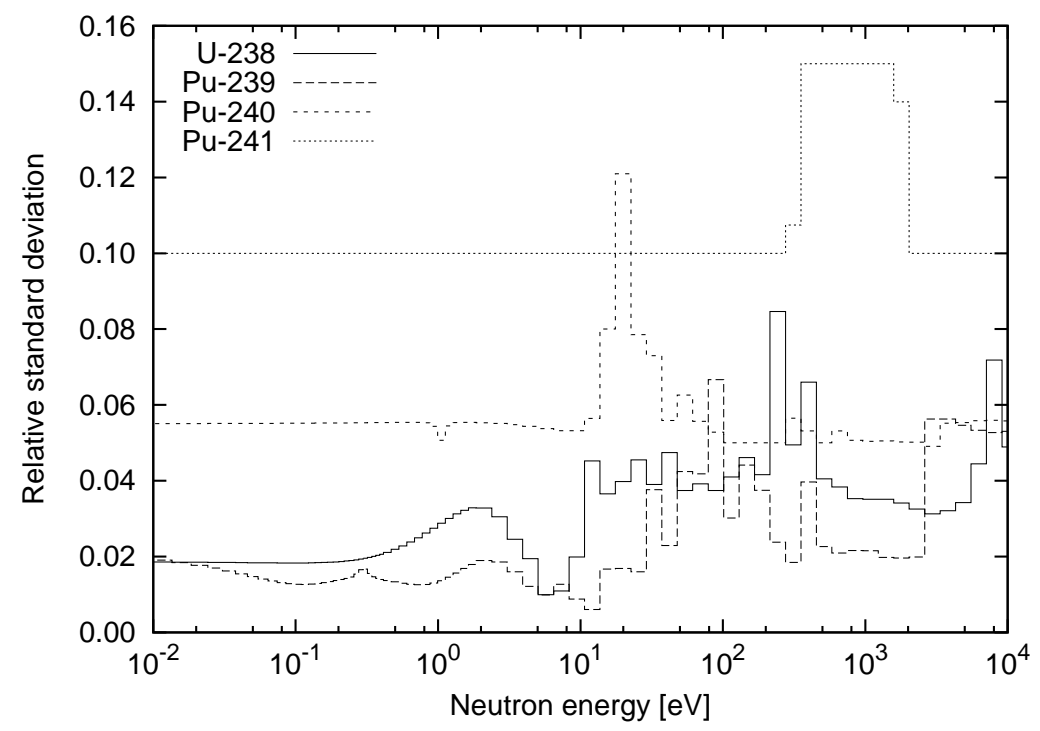

Figure 7 Relative standard deviations of capture cross sections in JENDL-4.0

G.Chiba:

Uncertainty quantification of neutronic parameters of light water reactor fuel cells with JENDL-4.0 covariance data 


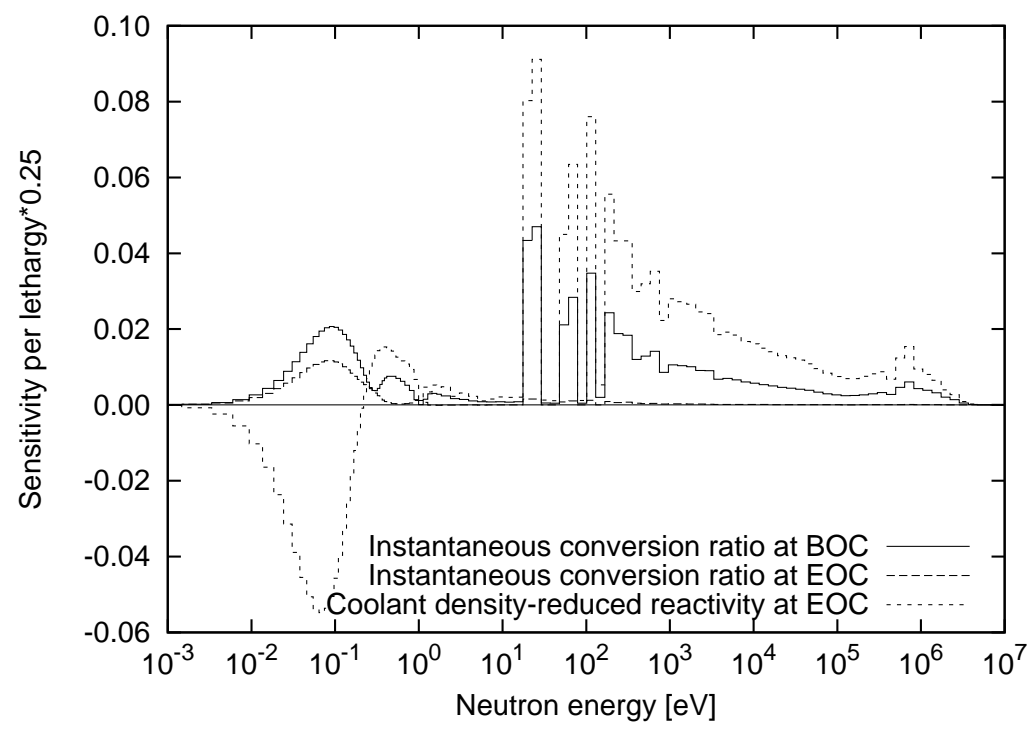

Figure 8 Sensitivity of neutronic parameters in Th-Pu cell

G.Chiba:

Uncertainty quantification of neutronic parameters of light water reactor fuel cells with JENDL-4.0 covariance data 